\title{
Germanica
}

Le goût des métamorphoses à propos de Die Letzte Welt de Christoph Ransmayr et de Der junge Mann de Botho Strauß

Die offene Verwandlung: Die letzte Welt Christoph Ransmayr und Der junge Mann von Botho Strau $\beta$

\section{Arlette Camion}

\section{OpenEdition}

\section{Journals}

Édition électronique

URL : http://journals.openedition.org/germanica/2167

DOI : 10.4000/germanica.2167

ISSN : 2107-0784

\section{Éditeur}

Université de Lille

\section{Édition imprimée}

Date de publication : 31 décembre 1993

Pagination : $27-40$

ISSN : 0984-2632

Référence électronique

Arlette Camion, «Le goût des métamorphoses à propos de Die Letzte Welt de Christoph Ransmayr et de Der junge Mann de Botho Strauß», Germanica [En ligne], 13 | 1993, mis en ligne le 20 septembre 2013, consulté le 06 octobre 2020. URL : http://journals.openedition.org/germanica/2167 ; DOI : https://doi.org/10.4000/germanica.2167

Ce document a été généré automatiquement le 6 octobre 2020.

(c) Tous droits réservés 


\title{
Le goût des métamorphoses à propos de Die Letzte Welt de Christoph Ransmayr et de Der junge Mann de Botho Strauß
}

\author{
Die offene Verwandlung: Die letzte Welt Christoph Ransmayr und Der junge \\ Mann von Botho Strau $\beta$
}

Arlette Camion

Le roman contemporain a derrière lui un lourd poids à traîner: la tradition du réalisme. La peinture a pu, beaucoup plus facilement que la littérature, faire table rase, se débarrasser de l'obligation de mimésis. Handke le remarque dans sa recherche toujours plus périlleuse d'une écriture neuve, pour le récit le recommencement est de façon obligée une répétition, déjouer le sens qui toujours se met en place avec les mots est une tâche difficile. De l'un à l'autre, les stratégies sont différentes : Arno Schmidt par exemple choisit de rompre avec toute narration convenue, il centre son texte sur le refus, l'ancre dans la révolte, le noue sur sa propre violence. Thomas Bernhard exclut toute temporalité extérieure à son texte, le module selon ses propres rythmes, en fait une partition qui lui confère une autonomie parfaite. D'autres encore jouent sur la prolifération du texte qui se met à évoluer aux confins du fantastique, qui se génère luimême : c'est le cas de Grass, et, d'une autre manière, celui de Gerhard Köpf.

2 Les deux romans que nous voudrions rapprocher ici sont très différents dans le traitement du récit: Die letzte Welt raconte une histoire, et Der junge Mann s'y refuse. Pourtant, tous les deux, ils mettent en place une double stratégie commune : ils sont jeu avec la structure romanesque de deux façons. D'abord parce qu'ils choisissent d'emblée comme réfèrent non le réel, qui serait la perspective à l'horizon du texte, mais un autre texte, dans un effet de mise en abyme. Ce procédé n'est pas neuf, il faisait déjà tout le charme de Der kurze Brief zum langen Abschied de Handke. Il est intéressant d'une part parce qu'il fait du récit le reflet d'un autre, son double, son spectre : mais aussi parce qu'il résout magiquement le problème de la répétition elle est revendiquée comme 
constitutive de l'écriture, comme son mécanisme même : une parole ne vient s'inscrire que dans le creux d'une autre. La réplique, la reprise délivre le récit de sa responsabilité d'être signifiant en soi : il ne l'est que par procuration, et cette insouciance peut être légèreté ou dangereux suspens. Die letzte Welt comme Der junge Mann choisissent cette apesanteur. Mais d'autre part, et ceci est leur seconde stratégie, ces deux récits font de la métamorphose non seulement le sujet, ou le motif de leur développement, mais encore son principe même: le passage d'une forme à l'autre, le jeu plastique des apparences, la dissolution des certitudes de la perception dans l'infinie variation du visible; voire même dans l'infinie variation du dicible. La métamorphose n'est pas du domaine de la rationalité, mais du domaine du mythe. Le Mythe est ce qui transcende toute formulation définitive, ce qui suscite un discours inachevé, reproductible à l'infini dans des formulations toujours différentes. Le Mythe touche au fantastique et produit des monstres, tout comme il touche à la sagesse et produit du sens. Ligne de partage floue, lieu des incertitudes et des intuitions, le mythe est en quelque sorte irréductible au texte qui le dit, il le transcende et le fonde à la fois.

C'est sur ce sol très peu ferme que les deux romans qui nous occupent prennent appui. Die letzte Welt, paru en 1988, est le second roman de Christoph Ransmayr. Il avait publié en 1984 un livre qui connut un succès immédiat, Die Schrecken des Eises und der Finsternis. L'histoire en était déjà à la fois facilement repérable, et dédoublée dans un jeu de miroir : il s'agissait de la relation presque documentaire de la première expédition polaire autrichienne au pôle Nord. Ransmayr ajoutait à son texte des extraits de mémoires des explorateurs, des passages de leur carnet de bord. L'effet réaliste était évident ; mais en même temps, cette première histoire était reprise en une seconde : un certain Josef Mazzini, triestin vivant à Vienne, homme de plusieurs cultures et de plusieurs langues, se prenait de passion pour les récits de l'expédition et décidait de la refaire. Les hommes partis en 1872 sont revenus après de terribles épreuves deux ans plus tard, Josef Mazzini, le héros fictif de cette quête purement romanesque ne reviendra pas. Il se perd dans le grand Nord, et il se perd aussi dans la reproduction absurde de cette histoire qui ne lui appartient pas. Ransmayr dans ce premier roman produisait un temps romanesque tout à fait original : le lecteur était contraint de passer de la rétrospective au présent de l'aventure de Mazzini, ce présent étant luimême une reproduction d'un réel devenu récit. L'écrivain Ransmayr se donnait aussi le droit de raconter l'histoire en son nom, et par ce jeu de glissement de la perspective narrative et du temps du récit, celui-ci se trouvait dans un déséquilibre qui relativisait la réalité des faits rapportés.

Dans Die letzte Welt, cette esquive du récit devant ses obligations de signifier un lieu et un temps nettement discernables se retrouve, sous une autre forme. Le décalage est aussi produit par la référence à d'autres histoires, celles des mythes que rapportent Les Métamorphoses d'Ovide. Ces Métamorphoses, ceci est la fiction de Ransmayr, ont été perdues et le personnage romanesque, un certain Cotta, décide de se mettre à la recherche du texte et du poète lui-même. Car texte et poète ont disparu : Ovide a été banni de Rome, il séjourne dans ce dernier des mondes qu'est la ville de Tomes, Tomi, Constanza, ville aux multiples noms, ville aux multiples formes, aux bouches du Danube. Le lecteur cultivé se rappelle les Tristes où Ovide disait la déréliction de son exil. Là encore le renvoi à une réalité attestée est manifeste. Pourtant Cotta n'est pas seulement le personnage qui frayait à Rome dans l'entourage d'Ovide, avant sa disgrâce, admirateur du grand artiste, secrètement jaloux de sa liberté à l'égard du pouvoir, de son insouciance face aux nécessités du réel. Cotta est cela, et nous le voyons 
évoluer dans les milieux de la cour de l'empereur Auguste. Mais il est aussi l'un des nôtres, notre contemporain. Dans Die Schrecken des Eises und der Finsternis, le dédoublement $d u$ récit des faits incontestablement réels se faisait par le truchement de Josef Mazzini, par la reprise de l'histoire dans un temps qui la dédoublait. Ici, c'est la trouvaille de Ransmayr, Cotta est simultanément le héros de deux histoires superposées: ou plutôt, il est le héros d'une histoire où se superposent deux temps distincts, pour ne plus faire qu'un. Cotta vit dans le monde antique, est au courant des affaires de Rome, mais il est aussi celui dont la mémoire est encombrée d'histoires de génocides, de tout un passé qui est le nôtre, à nous lecteurs, de toute une mémoire à la fois proche, moderne, et obscure, une mémoire faite d'images floues et effrayantes : fascination des foules pour l'orateur fou derrière les micros, horreur simplement entrevue des persécutions et des anéantissements muets. C'est dans cet univers factice (et non pas imaginaire), construit (et non pas rêvé) que se déroule le récit des événements : Die letzte Welt est presqu'autant roman d'aventures que Die Schrecken des Eises und der Finsternis. Il ne s'agit pas d'atteindre ici le pôle mythique, mais de retrouver le Texte mythique, qui exerce une fascination tout aussi forte. Un moment, Cotta le croit brûlé par le poète lui-même avant son départ, pour se soustraire aux trop réelles conséquences de la fiction ; peut-être est-il égaré, ou peut-être encore n'a-t-il jamais été écrit, peut-être est-il resté à l'état de fragments, épars, et dont Cotta ne pourra jamais recueillir que d'improbables traces. Tout ceci reste dit, et la réalité du texte d'Ovide au fond a moins d'importance que la quête qu'il ouvre. Le Texte de référence, comme une sorte d'objet qui se dérobe, comme la Forme qui ne se donne pas et abandonne le monde au chaos de l'Informe, demeure à l'horizon de l'incessante recherche de Cotta. Car même absent, il est essentiel : il est premier, il est modèle, il serait, s'il pouvait en disposer, pour Cotta la clef de tous les mystères. Mais le texte et Ovide lui-même demeurent introuvables. Publius Ovidius Naso, l'homme au grand nez, entre tous reconnaissable, a disparu: il n'est plus dans sa ferme ruinée de Trachila, dans les montagnes au dessus de Tomi, et pourtant son serviteur Pythagoras remplit comme à l'accoutumée la maison de victuailles; ce n'est pas lui, ce masque entrevu dans le brouillard d'un petit matin de carnaval, et pourtant toute la ville résonne de ses histoires. Sa présence est partout sensible : elle préside aux brusques changements des saisons, au passage sans transition de la tempête à l'immobilité de la canicule (chapitre 6), elle change le paysage dans les séismes (chapitre 11), elle inspire les récits d'Echo (chapitre 7), elle dicte à Arachné les histoires qu'illustrent ses tapisseries (chapitre 8). Ovide ne peut pas ne pas être là, et Cotta poursuit ses investigations. En chemin, il trouve des traces du Texte, bizarrement mêlées aux choses de la nature : des mots gravés sur des pierres, que Pythagoras doit d'abord débarrasser des mollusques qui y pullulent, quelques paroles inscrites sur des bouts d'étoffe flottant au vent. L'une d'elles vaut pour toutes: Keinem bleibt seine Gestalt (p.15). La métamorphose contamine en effet tout alentour : les habitants de Tomi se changent mystérieusement en pierre ou en bête, Echo passe de la laideur à la beauté la plus éclatante, les mûriers fleurissent dans la neige. Tous ces changements à vue, toutes ses étrangetés ne seraient que de l'ordre du fantastique, si elles n'étaient en quelque sorte l'illustration du Texte, son ombre portée. L'histoire de Ransmayr, parce qu'elle se donne sciemment comme une histoire de seconde main, échappe au merveilleux pour n'être que le redoublement de la fiction.

5 Ransmayr prend soin de donner à la fin de son roman un petit glossaire ovidien qui met en regard les histoires du poète latin et les siennes : ce n'est bien sûr pas par pur souci 
d'érudition. C'est au contraire pour bien faire apparaître combien son récit à lui est une sorte de double fantomatique du poème d'Ovide, en est une métamorphose. Il est une répétition hésitante, comme empêchée par le poids de mémoire accumulée, du texte original. Et en même temps sa transformation, car le texte perdu a été absorbé par la vie elle-même, les histoires d'Ovide sont devenues «les destins, les légendes, les rumeurs de cette côte-là » (p. 269): le boucher, l'épicière et son fils, l'amante de rencontre, le logeur sont de Tomi, la ville de fer, où Ransmayr, pense-t-on, a dû faire des repérages, tant le lieu a de présence, et ils sont simultanément les personnages d'Ovide. Ils ne sont donc ni vrais, ni faux, mais, comme l'ensemble de la construction du roman de Ransmayr, factices. Cette facticité se retrouve dans la métaphore du cinéma qui occupe tout le second chapitre : le lecteur assiste à une scène dans laquelle il a du mal à distinguer les plans constitutifs. On projette, un soir d'avril, sur le mur de la boucherie, un film en plein air ; le film montre la métamorphose de Ceyx et d'Alcyon. Toute la scène met en place un espace autre, qui n'est ni celui des aventures qui se déroulent sur l'écran, ni celui des spectateurs et de leurs réactions bruyantes, ni même le point de vue d'un narrateur extérieur: la perspective est faite d'un décalage savant de ces trois niveaux l'un par rapport à l'autre, de leur superposition, ou plutôt, pour reprendre un terme de cinéma, d'un fondu-enchaîné. Un espace naît d'un autre, comme une image naît d'une autre, après un court instant de superposition. D'une façon générale, le roman de Ransmayr semble, pour le lecteur, emprunter beaucoup à l'esthétique du film : la visualité y est très grande, comme le sens d'une spatialité vécue par le corps, et pourtant les images, nettes, semblent flotter, incertaines de leur origine et ignorantes de leur destination, irréelles. Le lecteur pourrait dire comme Cotta :

In diesem Gebirge verhallte die Welt, und Cotta erinnerte sich an sie. Wie die Luftblasen aus der Wassertiefe nach oben torkeln und steigen, so stiegen aus seinem Inneren Bilder auf, aus der Vergessenheit, und wurden, endlich oben, wieder zu nichts, (p. 42)

Ainsi Tomi apparaitt-elle comme un lieu terriblement là, sensible dans ses éléments, dans sa terre, son eau, son air, son feu, mais aussi comme un non-lieu, qui reste décalé de tout, un bout de terre entre les falaises, la mer et le fleuve, l'endroit du bannissement et de l'exil, où personne ne naît, où l'on ne fait qu'échouer. C'est là que se prolonge une attente qui a perdu tout sens (chapitre 13) ; c'est moins un refuge qu'un lieu ouvert à tous les périls, à tous les excès, à toutes les folies. Loin du pouvoir, mais aussi loin de la communauté raisonnable des hommes, la ville est semblable à celle que filme Angelopoulos dans Le pas suspendu de la cigogne: le dernier des mondes, celui qui n'a d'existence qu'à être l'endroit où l'on attend l'apocalypse.

Pourtant cette apocalypse ne va pas se produire. Elle aurait satisfait à la destination ordinaire du temps romanesque, qui est linéaire et qui ne peut aboutir que dans la rédemption ou la chute, dans la réconciliation, l'apaisement harmonieux des conflits, ou dans la mort ${ }^{1}$. Ransmayr échappe à cette fatalité en mettant en place deux catégories temporelles particulières: le temps de la recherche de Cotta ne progresse pas, c'est le temps cyclique des saisons, de l'éternel retour du Même, de l'absence de dénouement. Le temps du livre lui-même est un temps autre, ni avenir, ni présent, ni passé antique, mais un mélange répété des trois, une sorte d'anachronie qui ne peut être que celle de l'œuvre d'art elle-même ${ }^{2}$; le roman de cette manière parvient à se soustraire à la loi de la Durée qui régit habituellement toute narration. Le temps du roman est devenu d'une part le temps du mythe, où il y a un destin, mais pas de devenir (Cotta accomplit son destin en étant le fantôme qui hante Tomi), et d'autre part cette 
chose hybride, factice qui joue sur la métamorphose continue, sur le passage entre présent, passé, futur, sur le jeu de leurs croisements.

Il est peut-être intéressant d'insister à propos de Die letzte Welt de Ransmayr sur cette notion de facticité qui apparait, adjointe au principe de la métamorphose, être ce qui porte l'ensemble de la construction constituée par le livre. Trois facteurs y pourvoient, nous venons de le constater : l'intertextualité, la reprise d'un texte dans le texte, le procédé de mise en abyme ; l'invention d'une spatialité décalée, entre prégnance du lieu et évanescence du non-lieu; la mise en place d'une anachronie systématique, qui déjoue la linéarité romanesque. L'anachronie n'est pas éclatement du temps, car alors, comme il arrive souvent avec les techniques du Nouveau Roman français, le lecteur pourrait très bien reconstituer l'histoire a posteriori ; elle est plutôt esquive définitive dans une a-temporalité qui dispense le roman de désigner la réalité des choses et de délivrer le sens ultime. Apocalypse signifie la fin du secret, dit Baudrillard, et la fiction romanesque n'est jamais tant simulation, ni même mimésis que désir de venir à bout des choses et au bout du temps. Die letzte Welt au contraire, par une sorte d'ironie, se dérobe à l'ultime, à la fin du secret, à l'épuisement de la métaphore. Rien n'a été dit de plus que la répétition du mythe, qui n'explique rien, qui n'est l'indice de rien, mais qui se contente de raconter sans assigner de signification définitive à ce qui a été raconté. Grâce au principe de facticité, le secret est gardé quant à la vérité ou à la réalité des choses, mieux même, le secret est inclus dans le dire lui-même; comme le voit Baudrillard:

Il y a en fait une possibilité de se dégager de la réalité des choses, de leur sens et donc de leur apocalypse - car il est clair que le principe linéaire de réalité mène forcément à l'apocalypse, à la fin, il y a forcément la fin. Tandis qu'un principe qui est différent, un principe de facticité, c'est autre chose, on s'en dégage puisque même l'apocalypse entre comme un élément ésotérique en quelque sorte ${ }^{3}$.

Ainsi la facticité redouble-t-elle la fiction, elle la rend autonome, la délivre de la triste obligation de reproduire et d'interpréter: le roman, au lieu de tenter à toute force d'entraîner l'adhésion à la fiction qu'il met en place, au contraire va y faire obstacle, et ceci non en mettant en doute ce qu'il raconte, mais simplement en le mettant en suspens.

Par de nombreux côtés la facticité caractéristique aussi le roman de Botho Strauß Der junge Mann. Le recours à Pintertextualité est aussi présent, bien que caché: Ransmayr nomme son hypotexte, les Métamorphoses d'Ovide ; Strauß reprend le schéma du Wilhelm Meister de Goethe sans le nommer. Des éléments reviennent: la vocation théâtrale de Leo Pracht, la société de la Tour et l'évocation d'un plan pédagogique, la problématique de l'insertion de l'individu dans la société. Mais c'est surtout la tournure générale du récit qui rappelle le grand Bildungsroman goethéen: la narration s'organise en une Rahmenerzählung, qui occupe le premier et le dernier chapitre, et en une série de changements de décor, de ton, de ruptures du récit. Ici s'insère un conte fantastique, une histoire de sombres métamorphoses (Die Händlerin auf der hohen Kante, Die Frau meines Bruders), là un tableau vivant (Der stehende Liebespfeil), là une réflexion (Nur noch lichte Momente). L'impression de foisonnement domine celui de progression et ce que disait Goethe de son Wilhelm Meister pourrait très bien s'appliquer à Der junge Mann :

Mit solchem Büchlein aber ist es wie mit dem Leben selbst: es findet sich in dem

Komplex des Ganzen Notwendiges und Zufälliges; Vorgesetztes und

Angeschlossenes, bald gelungen, bald vereitelt, wodurch es eine Art von 
Unendlichkeit erhält, die sich in verständige und vernünftige Worte nicht durchaus fassen und einschliessen läßt ${ }^{4}$.

Et Strauß fait dire à l'acteur Ossia, dans le livre :

Jede Geschichte ist ein frevelhafter Eingriff in die schöpferische Unordnung des Leben... Die einzige Ausdrucksform, die der Wahrheit nahekommt: ein Haufen Zeugs.

Mais, dira-t-on, le principe de facticité que l'intertextualité produit, est annulé par la nature même du modèle, par le foisonnement du texte dans le Wilhelm Meister que Strauß ici reprend. Der junge Mann chercherait donc, comme l'avait fait le Bildungsroman de Goethe, à imiter la vie et non à redoubler la fiction. On pourrait le penser d'abord, mais le lecteur, lorsqu'il repose le livre a une impression bien différente. Car ce n'est pas la vie elle-même qu'il a vue dépeinte dans Der junge Mann, ce sont les apodes de la réflexion sur la vie. Lorsque Goethe varie la forme romanesque, lorsqu'il fait du roman un récit protéiforme, lorsqu'il mêle, dans le goût d'ailleurs du premier romantisme, maximes et action, essai et poésie, aphorisme et intrigue, le résultat qu'il obtient est celui de l'accumulation: car la vie est accumulatrice, proliférante, et d'autre part, et en même temps, une, unique, prise dans le processus de sa propre progression. Tous les épisodes, même ceux qui ralentissent le développement romanesque, ne sont que d'autres moyens épiques, d'autres événements dans le flot incessant et merveilleux du vivant, dans le ballet de ses formes et de ses transfigurations. La direction générale de l'entreprise toutefois reste claire : elle prend sens dans une utopie qui demeure à l'horizon du récit. Chez Strauß au contraire la prolifération des formes romanesques ne s'organise pas en un faisceau. Lorsque l'écrivain interrompt le fil narratif, lorsqu'il reprend la technique de l'insert qu'aimait Goethe, il introduit non tant une illustration de son propos qu'une diversion. Comme le remarque Fritz Raddatz, les variations de ton constituent une émulation entre les différents plans qui ne se complètent pas, mais se concurrencent ${ }^{5}$ : tendance au Bildungsroman, enfilade des rêves et de leurs métamorphoses à vue, images allégoriques du conte, argumentation rigoureuse, et, en basse continue, cette tonalité particulière de la langue qui est reprise du romantisme. Car ces différents niveaux ne s'organisent nullement en une narration homodiégétique, en une vision subjective et vraie du monde. Rien ne se conclut, rien ne débouche sur un ensemble plus vaste, tout reste à l'état de fragments, qui ne peuvent même plus être interprétés. Ainsi l'histoire de ces métamorphoses cauchemardesques de l'épisode Die Händlerin auf der hohen Kante, se termine-t-elle par un essai d'interprétation qui reste totalement infructueux. L'expert dépêché donne la conclusion suivante :

Es wird sehr schwer sein, das Ganze dieses ungewöhnlichen Vorfalls zu verstehen. Wir sind durchaus in der Lage, fast sämtliche Motive und Einzelteile des Geschehens zu entschlüsseln und abzuklären... Was uns aber fehlt, ist die Fähigkeit zu erkennen, welcher Ordnung des Seins das Geschehen denn letzlich im Ganzen angehört, (p. 130)

La même perte d'un sens qui viendrait structurer les fragments en un ensemble est décrite dans le chapitre de science-fiction qui s'intitule Die Siedlung comme ce qui caractérise la société de mutants des Syks. C'est une communauté marginale, mais qui pourrait bien préfigurer notre société post-industrielle. Les Syks sont à la fois combinateurs et naïvement religieux: ils recherchent partout des lois, mais ils admettent très bien que ces lois soient mouvantes, car dit l'ethnologue qui les observe : 
Auch ihr ganzes Sozialgebilde war ja ein traumförmiges, und wurde beherrscht vom

Gesetz der offenen Verwandlung, (p. 132) n'est pas le sens, mais le retrait du sens dans le jeu des formes, la crise du sens. Hubert Winkels voit dans l'attention au détail, l'observation parfois très éclairante de notre quotidienneté, et le refus d'interprétation une marque de la "sensibilité fragmentée " de Botho Strauß; le texte ne veut plus qu'une chose : organiser, rassembler, associer ces moments sensibles en une texture ${ }^{6}$. Encore ne peut-on même pas dire que le texte trouve son unité dans l'unicité d'une seule voix narrative. Au contraire: on ne sait jamais qui y parle, qui l'on entend. L'énergie narrative n'est non plus pas égale, elle donne l'impression de se disperser, de se disséminer. Le texte est en fait constitué de l'incessante métamorphose d'une parole qui pourrait être devinée derrière lui. Joachim Kaiser se demande comment l'on pourrait la définir, et il l'imagine comme un Je auctorial à découvert, sans défense, sans stratégie de défense de sa propre identité, ressemblant de la sorte au Je que l'on entend dans les Minima Moralia d'Adorno ${ }^{7}$. Tout ce qui vient réduire ce Je, ce qui le contredit ou le contrecarre a droit à l'expression : les rêves et les visions, les formules incantatoires, les répétitions qui révèlent l'artifice, les parenthèses et les digressions.

On peut bien sûr évoquer ici la disparition du Sujet telle que l'illustrent les thèses de Foucault, et Fritz Raddatz fait le rapprochement entre la dissémination du Je narratif chez Botho Strauß et la constatation, à la fin de Les mots et les choses, de l'avènement d'un monde d'où le sujet a disparü. En effet, dans Der junge Mann, ce sont des sentiments qui viennent se heurter à d'autres sentiments, ou qui viennent se heurter à la raison. Mais cette disparition n'est ni tragique, ni comique. Elle est simplement, dit Strauß, intéressante: elle fait quitter les dimensions du sens, la profondeur et la hauteur, pour mettre en place un simple jeu des surfaces :

Trauern wir also nicht länger der verschollenen Tiefe, der verflüchtigten Höhe nach. Die komplexe Fläche zu erfahren ist keine mindere Leistung des menschlichen Geistes als seine Ausdehnung zu den Müttern nach unten und dem Vater nach oben. (p. 204)

Le projet faustien d'une expérience qui aille des Mères, des racines obscures, au Père, à la plus grande spiritualité, était un désir de totalité. Le fragment au contraire sousentend qu'une telle totalité ne peut être atteinte, voire même qu'elle n'existe tout simplement pas, car il n'y a pas d'instance unificatrice de l'expérience, ni sens, ni sujet. Le sujet, en admettant qu'il puisse encore se concevoir comme tel, n'est pas tant à la dérive, qu'à la découverte. La subjectivité est en constante mouvance, non pas identique à elle-même dans l'appréhension d'un univers mis en perspective par la ligne de son regard, mais changeante, passant d'un bout d'univers à un autre, se métamorphosant à chaque passage : dans les cinq chapitres qui constituent le roman, à chaque faisant une nouvelle expérience du monde, à chaque fois autre et comme reprenant à chaque fois la lecture du monde depuis le début. Bien évidemment, l'entreprise du Bildungsroman est alors impossible, celle d'une éducation, d'une lente maturation du Sujet dans sa compréhension de soi, du monde et des autres, dans l'harmonieuse résolution de tous les conflits que son indivuation avait fait naître. Der junge Mann est dans cette mesure un Bildungsroman en fin de parcours, en fin de partie, un Bildungsroman de la post-modernité qui ne croit plus aux Lumières, à la raison, à l'épanouissement de soi dans la rationalité.

Germanica, 13 | 1993 
17 Der junge Mann fait sienne la théorie des surfaces, des glissements, des transparences de Jean-François Lyotard: l'apparence devient l'essence, dans le règne de ces catégories que l'on nomme «immatérielles ». Le récit, parce qu'il ne saurait se satisfaire de la simple verticalité du fragment, est pris dans une dynamique, mais elle devient un pur flux, un « Fluxus », comme disait le plasticien Josef Beuys, transformation et dispersion d'énergie, au lieu de la totalisation du sens. L'harmonie, la «schöne Geselligkeit» goethéenne sont reprises dans tout le chapitre central du roman, intitulé La Terrasse. L'histoire de Jossica est aussi celle d'une intégration, d'un dépassement des conflits par l'insertion acceptée dans la communauté des autres. Mais cette belle trajectoire n'aboutit à rien de précis, elle n'est qu'un flux parmi d'autres, qui se perd dans le réseau compliqué des autres ramifications du récit. Hans-Josef Ortheil prétend que ce genre de livre finalement débouche dans une Totalité utopique ${ }^{9}$ : peut-être, mais alors utopie vide, où rien ne permet de rester. En fait, le roman n'intègre rien, ne totalise rien, il est, comme le montre Joachim Kaiser ${ }^{10}$ un roman sans centre, où épisodes, réflexions, observations restent à l'état de simples mouvements de pensée. La forme du Bildungsroman n'est plus qu'un alibi narratif, une forme reprise et variée, métamorphosée dans le désenchantement, devenu pur support, pur geste, extérieure. L'identité de soi à soi dans le rapport aux autres, le grand thème du Bildungsroman, ne fait plus le centre du récit, mais est diffracté en un faisceau mouvant d'histoires qui ne se rejoignent pas. Il arrive là ce qu'Adorno prévoyait, dans les Minima Moralia :

Die großen Themen werden verkommen, aber kaum im traditionnellen Sinn

«thematisch», sondern gebrochen und exzentrisch ${ }^{11}$.

18 Ainsi le principe de "métamorphose ouverte ", offene Verwandlung a-t-il remplacé celui de récit, de diégèse, et rendu caduque la recherche du sens. Il ne reste plus qu'un continuum de représentations, d'observations, d'images, que la raison ne retient plus ensemble et qui peuvent à tout moment verser dans le délire, l'épouvante, l'excès des visions sataniques. Les métamorphoses des choses dans le rêve, leur mouvante transformation, imprévisible et effrayante, voilà la matrice même de l'écriture de Botho Strauß. Or le rêve, dit-il, supprime l'objet, le fait disparaître en se l'appropriant, en le résolvant: «Der Traum schafft den Gegenstand ab. Er ist reine Einvernahme, Lösung» (p.27). La conscience de celui qui écrit est elle-même en perpétuelle métamorphose, absorbée par la fascination du Mythe, ici la sombre mythologie germanique, ou bien prise dans le jeu incessant de l'attirance et de la répulsion, du désir de scission ou de fusion. La métamorphose est pour Botho Strauß liée à cet Eros qui soutient le geste d'écrire, désir brutalement renvoyé à lui-même, jamais n'aboutissant, toujours renaissant:

Aller Stoff ist erotische Metamorphose. Das Gelüst selbst ist der Stoff, kurzlebig und sprunghaft.

... Die Geschichten der Verwandlung müssen eine Waschung sein, zur reinen Geschlechtlichkeit hin. (p. 368)

Die letzte Welt de Ransmayr et Der junge Mann de Botho Strauß ont remplacé le sens par l'énigme. Comme dans le Nom de la Rose d'Umberto Eco, l'interrogation reste ouverte, le labyrinthe n'a pas été encore complètement exploré, il demeure cette frange floue d'où pourrait venir d'autres métamorphoses encore. Cotta, comme le montre Jean-Charles Margotton, «croyait trouver des récits mythiques sous une forme objectivée, consommable par la raison, il trouve des choses que l'on ne peut détacher de la Vie ellemême, il n'y a pas de séparation entre les récits sur le monde et le monde lui-même ${ }^{12}$. Le jeune homme de Strauß n'est pas plus avancé à la fin du livre, il n'a rien appris. Et le 
roman lui-même n'aura été qu'une feinte. Der junge Mann et Die letzte Welt sont à double titre des récits "spectraux »: ils sont, pris dans le phénomène de la métamorphose, fantômes d'eux-mêmes, et, pris dans le reflet de la réplique, doubles d'eux-mêmes, multipliés dans leur propre image.

\section{NOTES}

1. - Voir les réflexions de Peter Handke à ce sujet, en particulier dans Die Lehre der Sainte-Victoire, Suhrkamp, 1980, p. 74-75.

2. - Voir à ce propos Daniel Payot, Anachronies de l'œuvre d'art, Galilée, 1990.

3. - Jean Baudrillard, Figures de Valtérité, Descartes, 1992, p. 63.

4. - Lettre de Goethe à Rochlitz, du 23.11.1829.

5. - Fritz Raddatz, Zur deutschen Literatur der Zeit, Rowohlt, 1987, p. 376.

6. - Hubert Winkels, Selbstheilung des Fragments, in Sprache im technischen Zeitalter, Januar, 1983, H. 85.

7. - Joachim Kaiser, Erlebte Literatur, Piper, 1988, p. 414.

8. - Fritz Raddatz, op. cit.

9. - Sur "l'effet Schéhérazade ", voir Jürgen Egyptien, Scheherazade, ewiger Umgang und Klandestinät, in Text und Kritik, $\mathrm{n}^{\circ} 113$.

10. - Joachim Kaiser, op. cit.

11. - Theodor Adorno, Minima Moralia, Suhrkamp, 1951, p. 83.

12. - Jean-Charles Margotton, L'écriture mythique dans Die Letzte Welt de Christoph Ransmayr, in Etudes Allemandes, Université de Lyon II, $\mathrm{n}^{\circ} 2$.

\section{RÉSUMÉS}

Le dernier des mondes de Christoph Ransmayr et Le jeune homme de Botho Strauß sont des romans post-modernes qui déjouent le réalisme en jouant sur Pintertextualité et se délivrent de l'obligation de signifier en privilégiant le fragmentaire. L'apocalypse, dans Le dernier des mondes, n'aura pas lieu, et le jeune homme ne deviendra jamais quelqu'un : rien ne se conclut, tout reste en suspens. Si Ransmayr reprend le texte des Métamorphoses d'Ovide, c'est en le transposant dans un temps et un lieu qui ne sont ni réels, ni vraiment fictifs, mais simplement factices. Si Botho Strauß reprend la thématique du grand Bildungsroman goethéen, c'est comme un simple alibi pour une écriture dont le principe n'est pas celui de la linéarité, ni de la progression vers plus de rationalité, mais celui de la « métamorphose ouverte ». L'écriture est le glissement d'une forme à l'autre, et la voix narrative se dissémine chez Strauß, se diffracte chez Ransmayr, produisant un texte qui se veut avant tout trace, non pas recherche du sens, mais proximité du Mythe. La facticité rend à la fiction son autonomie, le mythe lui rend son énigme, la métamorphose reste son seul principe dynamique. Evoluant dans un univers aux contours flous, Le dernier des mondes 
et Le jeune homme sont des récits "spectraux" à double titre: fantômes d'eux-mêmes et dédoublés dans leur propre image.

Die Postmoderne hat bekanntlich eine Vorliebe für das Fragmentarische und die Intertextualität. Die letzte Welt von Christoph Ransmayr und Der junge Mann von Botho Strauß sind postmoderne Romane, Erzählungen aus zweiter Hand, die sich der Verpflichtung der Totalität und der Sinngebung entledigen. Die letzte Welt ist keine Vision der Apokalypse, weil hier die Apokalypse als Variationsmodell mit in den Schreibprozeß selbst einbezogen wird; Der junge Mann ist kein neuer Bildungsroman, der in die Utopie der Selbstverwirklichung münden würde, sondern bloße Wiederholung von Themen, die, um mit Adorno zu sprechen, «exzentrich geworden sind». Die Suche nach Sinn ist einer Kunst der Oberfläche gewichen, deren Prinzip einerseits die «facticité» (Baudrillard) und andrerseits «die offene Verwandlung» (Botho Strauß) ist. Der junge Mann und Die letzte Welt entweichen den strengen Gesetzen der Rationalität, um sich dem Mythos hinzugeben; sie bewegen sich stets zwischen Traum und Wirklichkeit, in einer Welt, die keine festen Konturen mehr besitzt. Nicht nur thematisch, sondern auch in ihrem Wesen selbst sind diese beiden Romane gespensterhaft : schattenhafte Erzählungen, gleichsam von der eigenen Identität befreit.

\section{AUTEUR}

\section{ARLETTE CAMION}

Université Charles-de-Gaulle - Lille III 